\title{
358.
}

\section{ADDITION TO THE MEMOIR ON TSCHIRNHAUSEN'S TRANSFORMATION.}

[From the Philosoplical Transactions of the Royal Society of London, vol. CLvi. (for the year 1866), pp. 97-100. Received October 24,-Read December 7, 1866.]

IN the memoir "On Tschirnhausen's Transformation," Philosophical Transactions, vol. CLII. (1862), pp. 561-568, [275], I considered the case of a quartic equation: viz. it was shown that the equation

is, by the substitution

$$
\left(a, b, c, d, e^{\gamma} x, 1\right)^{4}=0
$$

$$
y=(a x+b) B+\left(a x^{2}+4 b x+3 c\right) C+\left(a x^{3}+4 b x^{2}+6 c x+3 d\right) D
$$

transformed into

$$
\left(1,0,(5, \mathfrak{D},(5) y, 1)^{4}=0\right.
$$

where $(\mathbb{E}, \mathfrak{D}, \mathbb{E})$ have certain given values. It was further remarked that $(\mathbb{E}, \mathfrak{D},(\mathbb{E})$ were expressible in terms of $U^{\prime}, H^{\prime}, \Phi^{\prime}$, invariants of the two forms $(a, b, c, d, e \gamma X, Y)^{4}$, $\left(B, C, D_{\chi} Y,-X\right)^{2}$, of $I, J$, the invariants of the first, and of $\Theta^{\prime},=B D-C^{2}$, the invariant of the second of these two forms, viz. that we have

$$
\begin{aligned}
& \mathfrak{E}=6 H^{\prime}-2 I \Theta^{\prime}, \\
& \mathfrak{D}=4 \Phi^{\prime}, \\
& \mathfrak{E}=I U^{\prime 3}-3 H^{\prime 2}+I^{2} \Theta^{\prime 2}+12 J^{\prime} \Theta^{\prime} U^{\prime}+2 I^{\prime} \Theta^{\prime} H^{\prime} ;
\end{aligned}
$$

and by means of these I obtained an expression for the quadrinvariant of the form

$$
\left(1,0, \mathfrak{5}, \mathfrak{D},(\varepsilon \gamma y, 1)^{4}\right. \text {; }
$$

viz. this was found to be

$$
=I U^{\prime 2}+\frac{4}{3} I^{2} \Theta^{\prime 2}+12 J \Theta^{\prime} U^{\prime}
$$

C. V. 
But I did not obtain an expression for the cubinvariant of the same function: such expression, it was remarked, would contain the square of the invariant $\Phi^{\prime}$; it was probable that there existed an identical equation,

$$
J U^{\prime 3}-I U^{\prime 2} H^{\prime}+4 H^{\prime 3}+M \Theta^{\prime}=-\Phi^{\prime 2},
$$

which would serve to express $\Phi^{\prime 2}$ in terms of the other invariants; but, assuming that such an equation existed, the form of the factor $M$ remained to be ascertained; and until this was done, the expression for the cubinvariant could not be obtained in its most simple form. I have recently verified the existence of the identical equation just referred to, and have obtained the expression for the factor $M$; and with the assistance of this identical equation I have obtained the expression for the cubinvariant of the form

$$
\left(1,0,(5, \mathfrak{D},(\xi) y, 1)^{4} .\right.
$$

The expression for the quadrinvariant was, as already mentioned, given in the former memoir: I find that the two invariants are in fact the invariants of a certain linear function of $U, H$; viz. the linear function is $=U^{\prime} U+\frac{2}{3} \Theta^{\prime} H$; so that, denoting by $I^{*}$, $J^{*}$, the quadrinvariant and the cubinvariant respectively of the form

we have

$$
\left(1,0,\left(5, \mathfrak{D},(\xi \gamma y, 1)^{4},\right.\right.
$$

$$
\begin{aligned}
& I^{*}=\tilde{I}\left(U^{\prime} U+4 \Theta^{\prime} H\right), \\
& J^{*}=\tilde{J}\left(U^{\prime} U+4 \Theta^{\prime} H\right),
\end{aligned}
$$

where $\tilde{I}, \tilde{J}$ signify the functional operations of forming the two invariants respectively. The function $\left(1,0, \mathfrak{E}, \mathcal{D},(\mathfrak{E} \& y, 1)^{4}\right.$, obtained by the application of Tschirnhausen's transformation to the equation

$$
\left(a, b, c, d, e \gamma(x, 1)^{4}=0,\right.
$$

has thus the same invariants with the function

$U^{\prime} U+4 \Theta^{\prime} H=U^{\prime}\left(a, b, c, d, e \gamma(x, 1)^{4}+4 \Theta^{\prime}\left(a c-b^{2}, a d-b c, a e+2 b d-3 c^{2}, b e-c d, c e-d^{2} \gamma(x, 1)^{4}\right.\right.$,

and it is consequently a linear transformation of the last-mentioned function; so that the application of Tschirnhausen's transformation to the equation $U=0$ gives an equation linearly transformable into, and thus virtually equivalent to, the equation

$$
U^{\prime} U+4 \Theta^{\prime} H=0,
$$

which is an equation involving the single parameter $\frac{4 \Theta^{\prime}}{U^{\prime}}$ : this appears to me a result of considerable interest. It is to be remarked that Tschirnhausen's transformation, wherein $y$ is put equal to a rational and integral function of the order $n-1$ (if $n$ be the order of the equation in $x$ ), is not really less general than the transformation wherein $y$ is put equal to any rational function $\frac{V}{W}$ whatever of $x$; such rational function may, in fact, by means of the given equation in $x$, be reduced to a rational and integral function of the order $n-1$; hence in the present case, taking $V, W$ to 
be respectively of the order $n-1,=3$, it follows that the equation in $y$ obtained by the elimination of $x$ from the equations

$$
\begin{gathered}
\left(a, b, c, d, e \gamma(x, 1)^{4}=0,\right. \\
y=\frac{\left(\alpha, \beta, \gamma, \delta \gamma(x, 1)^{3}\right.}{\left(\alpha^{\prime}, \beta^{\prime}, \gamma^{\prime}, \delta^{\prime}(x, 1)^{3}\right.},
\end{gathered}
$$

is a mere linear transformation of the equation $A U+B H=0$, where $A, B$ are functions (not as yet calculated) of ( $\left.a, b, c, d, e, \alpha, \beta, \gamma, \delta, \alpha^{\prime}, \beta^{\prime}, \gamma^{\prime}, \delta^{\prime}\right)$.

Article Nos. 1, 2, 3. Investigation of the identical equation

$$
J U^{\prime 3}-I U^{\prime 2} H^{\prime}+4 H^{\prime 3}+M \Theta^{\prime}=-\Phi^{\prime 2} \text {. }
$$

1. It is only necessary to show that we have such an equation, $M$ being an invariant, in the particular case $a=e=1, b=d=0, c=\theta$, that is for the quartic function $\left(1,0, \theta, 0,1 \gamma(x, 1)^{4}\right.$; for, this being so, the equation will be true in general. Writing the equation in the form

$$
-M \Theta^{\prime}=U^{\prime 2}\left(J U^{\prime}-I H^{\prime}\right)+4 H^{\prime 3}+\Phi^{\prime 2},
$$

and observing that we have

$$
\begin{aligned}
& U^{\prime}=\left(B^{2}+D^{2}\right)+2 \theta B D+4 \theta C^{2}, \\
& H^{\prime}=\theta\left(B^{2}+D^{2}\right)+\left(1+\theta^{2}\right) B D-4 \theta^{2} C^{2}, \\
& \Theta^{\prime}=B D-C^{2} \\
& \Phi^{\prime}=\left(1-9 \theta^{2}\right) C\left(B^{2}-D^{2}\right) \\
& I=1+3 \theta^{2} \\
& J=\theta-\theta^{3}
\end{aligned}
$$

and thence

$$
J U^{\prime}-I H^{\prime}=-4 \theta^{3}\left(b^{2}+D^{2}\right)+\left(-1-2 \theta^{2}-\tilde{5} \theta^{4}\right) B D+\left(8 \theta^{2}+8 \theta^{4}\right) C^{2},
$$

the equation becomes

$$
\begin{aligned}
&-\left(B D-C^{2}\right) M= \\
& \quad\left\{-4 \theta^{3}\left(B^{2}+D^{2}\right)+\left(-1-2 \theta^{2}-5 \theta^{4}\right) B D+\left(8 \theta^{2}+8 \theta^{4}\right) C^{2}\right\} \times\left\{B^{2}+D^{2}+2 \theta B D+4 \theta C^{2}\right\}^{2} \\
&+ 4\left\{\theta\left(B^{2}+D^{2}\right)+\left(1+\theta^{2}\right) B D-4 \theta^{2} C^{2}\right\}^{3} \\
&+\left(1-9 \theta^{2}\right)^{2} C^{2}\left\{\left(B^{2}+D^{2}\right)^{2}-4 B^{2} D^{2}\right\} .
\end{aligned}
$$


2. It is found by developing that the right-hand side is in fact divisible by $B D-C^{2}$, and that the quotient is

$$
\begin{aligned}
= & \left(-1+10 \theta^{2}-9 \theta^{4}\right)\left(B^{2}+D^{2}\right)^{2} \\
+ & \left(8 \theta+16 \theta^{3}-24 \theta^{5}\right)\left(B^{2}+D^{2}\right) B D \\
+ & \left(4+8 \theta^{2}+4 \theta^{4}-16 \theta^{6}\right) B^{2} D^{2} \\
+ & \left(-64 \theta^{3}-192 \theta^{5}\right)\left(B^{2}+D^{2}\right) C^{2} \\
+ & \left(16 \theta^{2}-416 \theta^{4}-112 \theta^{6}\right) B D C^{2} \\
+ & \left(-128 \theta^{4}+128 \theta^{6}\right) C^{4} .
\end{aligned}
$$

3. This is found to be

$$
\begin{aligned}
= & -I^{2} U^{\prime 2}+12 J U^{\prime} H^{\prime}+4 I H^{\prime 2} \\
& -8 I J U^{\prime} \Theta^{\prime} \\
& -16 J^{2} \Theta^{\prime 2},
\end{aligned}
$$

which is consequently the value of $-M$. We have therefore

$$
\begin{aligned}
-\Phi^{\prime 2}= & J U^{\prime 3}-I U^{\prime 2} H^{\prime}+4 H^{\prime 3} \\
& +\left(I^{2} U^{\prime 2}-12 J U^{\prime} H^{\prime}-4 I H^{\prime 2}\right) \Theta^{\prime} \\
& +8 I J U^{\prime} \Theta^{\prime 2} \\
& +16 J^{2} \Theta^{\prime 3}
\end{aligned}
$$

which is the required identical equation.

Article No. 4. Calculation of the Cubinvariant.

4. We have

$$
\begin{aligned}
J^{*}= & \frac{1}{6}\left(\widetilde { E } \cdot \left(\xi-\left(\frac{1}{6}(5)^{3}-\left(\frac{1}{4} D\right)^{2}\right.\right.\right. \\
= & \left(H-\frac{1}{3} I \Theta^{\prime}\right)\left\{I U^{\prime 2}-3 H^{\prime 2}+\left(12 J U^{\prime}+2 I H^{\prime}\right) \Theta^{\prime}+I^{2} \Theta^{\prime 2}\right\} \\
& -\left(H-\frac{1}{3} I \Theta^{\prime}\right)^{3} \\
& -\Phi^{\prime 2},
\end{aligned}
$$

whence, substituting for $-\Phi^{\prime 2}$ its value and reducing, we find

$$
J^{*}=J U^{\prime 3}+\Theta^{\prime} \cdot \frac{2}{3} I^{2} U^{\prime 2}+\Theta^{\prime 2}\left(4 I J U^{\prime}\right)+\Theta^{\prime 3}\left(16 J^{2}-\frac{8}{27} I^{3}\right) .
$$

Article No. 5. Final expressions of the two Invariants.

The value of $I^{*}$ has been already mentioned to be $I^{*}=I U^{\prime 2}+\Theta^{\prime} 12 J U^{\prime}+\Theta^{\prime 2} \cdot \frac{4}{3} I^{2}$, and it hence appears that the values of the two invariants may be written

$$
\begin{aligned}
& I^{*}=\left(I, 18 J, 3 I^{2} \gamma U^{\prime}, \frac{2}{3} \Theta^{\prime}\right)^{2}, \\
& J^{*}=\left(J, I^{2}, 9 I J,-I^{3}+54 J^{2} \gamma U^{\prime}, \frac{2}{3} \Theta^{\prime}\right)^{3} .
\end{aligned}
$$


But we have (see Table No. 72 in my "Seventh Memoir on Quantics," Philosophical Transactions, vol. CLI. (1861), pp. 277—292, [269])

$$
\begin{aligned}
& \tilde{I}(\alpha U+6 \beta H)=\left(I, 18 J, 3 I^{\gamma}(\alpha, \beta)^{2},\right. \\
& \tilde{J}(\alpha U+6 \beta H)=\left(J, I^{2}, 9 I J,-I^{3}+54 J^{2} \gamma(\alpha, \beta)^{3} ;\right.
\end{aligned}
$$

so that, writing $\alpha=U^{\prime}, \beta=\frac{2}{3} \Theta^{\prime}$, we have

$$
\begin{aligned}
& I^{*}=\widetilde{I}\left(U^{\prime} U+4 \Theta^{\prime} H\right), \\
& J^{*}=\widetilde{J}\left(U^{\prime} U+4 \Theta^{\prime} H\right) ;
\end{aligned}
$$

or the function $\left(1,0,(5, D),(\varepsilon \gamma y, 1)^{4}\right.$ obtained from Tschirnhausen's transformation of the equation $U=0$ has the same invariants with the function $U^{\prime} U+4 \Theta^{\prime} H$; or, what is the same thing, the equation $\left(1,0, \mathfrak{E}, \mathcal{D},(\mathfrak{E} y y, 1)^{4}=0\right.$ is a mere linear transformation of the equation $U^{\prime} U+4 \Theta H=0$; which is the above-mentioned theorem. 